\title{
Activation of the RpoN-RpoS regulatory pathway during the enzootic life cycle of Borrelia burgdorferi
}

\author{
Zhiming Ouyang ${ }^{1}$, Sukanya Narasimhan², Girish Neelakanta², Manish Kumar ${ }^{3}$, Utpal Pal $^{3}$, Erol Fikrig ${ }^{2}$ and \\ Michael $V$ Norgard ${ }^{1 *}$
}

\begin{abstract}
Background: The maintenance of Borrelia burgdorferi in its complex tick-mammalian enzootic life cycle is dependent on the organism's adaptation to its diverse niches. To this end, the RpoN-RpoS regulatory pathway in $B$. burgdorferi plays a central role in microbial survival and Lyme disease pathogenesis by up- or down-regulating the expression of a number of virulence-associated outer membrane lipoproteins in response to key environmental stimuli. Whereas a number of studies have reported on the expression of RpoS and its target genes, a more comprehensive understanding of when activation of the RpoN-RpoS pathway occurs, and when induction of the pathway is most relevant to specific stage(s) in the life cycle of B. burgdorferi, has been lacking.

Results: Herein, we examined the expression of rpoS and key lipoprotein genes regulated by RpoS, including ospC, ospA, and $d b p A$, throughout the entire tick-mammal infectious cycle of B. burgdorferi. Our data revealed that transcription of rpoS, OspC, and $d b p A$ is highly induced in nymphal ticks when taking a blood meal. The RpoN-RpoS pathway remains active during the mammalian infection phase, as indicated by the sustained transcription of rpoS and $d b p A$ in B. burgdorferi within mouse tissues following borrelial dissemination. However, dbpA transcription levels in fed larvae and intermolt larvae suggested that an additional layer of control likely is involved in the expression of the $d b p B A$ operon. Our results also provide further evidence for the downregulation of ospA expression during mammalian infection, and the repression of ospC at later phases of mammalian infection by $B$. burgdorferi.
\end{abstract}

Conclusion: Our study demonstrates that the RpoN-RpoS regulatory pathway is initially activated during the tick transmission of $B$. burgdorferi to its mammalian host, and is sustained during mammalian infection.

\section{Background}

Lyme borreliosis, caused by the spirochetal bacterium Borrelia burgdorferi, remains the most common vectorborne disease in the United States [1]. B. burgdorferi is transmitted either to its natural mammalian host(s) or inadvertently to humans through the bite of an infected Ixodes tick vector [2,3]. In humans, B. burgdorferi causes a localized skin lesion (erythema migrans) at the initial site of infection, followed by hematogenous dissemination of the bacterium to distant sites such as the heart,

\footnotetext{
* Correspondence: michael.norgard@utsouthwestern.edu

'Department of Microbiology, University of Texas Southwestern Medical

Center, 5323 Harry Hines Blvd, Dallas, TX 75390, USA Full list of author information is available at the end of the article
}

joints, and central nervous system, causing carditis, arthritis, and neurological manifestations [1-3].

To maintain itself in its complex tick-mammalian infectious life cycle, B. burgdorferi must adapt to two markedly different host milieus (ticks and mammals). This host adaptation is achieved, at least in part, by altering a number of its outer surface lipoproteins, which is perhaps best exemplified by the differential regulation of outer surface (lipo)protein A (OspA) and outer surface (lipo)protein C (OspC) [4-9]. OspA, serving as an attachment factor for the tick midgut protein TROSPA, is important for B. burgdorferi to colonize and survive in tick midguts [10-12]. OspC, although its precise function remains unknown, is essential for B. burgdorferi to establish itself in the mammalian setting,
C Biomed Central 
particularly at the early stage of infection [13-15]. As such, in flat (unfed) nymphs, OspA, but not OspC, is abundantly expressed on the surface of spirochetes, whereas during early mammalian infection, OspC, but not OspA, is highly induced [4,7-9].

There is now compelling evidence that the differential regulation of $\operatorname{ssp} C$ and other outer membrane lipoproteins in $B$. burgdorferi is mediated by a central regulatory cascade known as the RpoN-RpoS regulatory pathway [16-21]. In the RpoN-RpoS pathway, one alternative sigma factor (sigmaN, $\sigma^{\mathrm{N}}, \sigma^{54}$, RpoN) controls the expression of another alternative sigma factor (sig$\mathrm{maS}, \sigma^{\mathrm{s}}, \sigma^{38}, \mathrm{RpoS}$ ) which, in turn, governs the expression of key membrane lipoproteins associated with borrelial virulence. Like other bacterial $\sigma^{54}$-dependent systems, activation of $B$. burgdorferi rpoS requires a putative enhancer-binding protein (EBP), Rrp2, which has been postulated to be activated through phosphorylation [22-26]. However, unlike most other bacterial EBPs for $\sigma^{54}$ systems, Rrp2 has been reported not to bind specifically to DNA region(s) in proximity to the $\sigma^{54}$-dependent rpoS promoter in B. burgdorferi [23,27]. Surprisingly, another activator, BosR, recently has been shown to be an additional molecule that also is essential for $\sigma^{54}$-dependent rpoS transcription in B. burgdorferi [21,28-31]; data thus far suggest that BosR binds to one or more sites near the rpoS promoter through a novel DNA binding mechanism [30]. Finally, rpoS expression also is modulated by the small RNA DsrA (and its potential chaperone $\mathrm{Hfq}$ ) [32,33], CsrA (the putative carbon storage regulator A) [34,35], and other unknown mammalian host factors [17,21,36-38].

Under in vitro culture parameters of lower temperature $\left(23^{\circ} \mathrm{C}\right)$ and a Barbour-Stoenner-Kelly (BSK) medium $\mathrm{pH}$ of about 7.4, conditions that ostensibly mimic those of the unfed tick midgut, the expression of rpoS in B. burgdorferi is repressed. Changes in these environmental conditions emanating from the tick's taking of a blood meal, such as elevated temperature $\left(37^{\circ} \mathrm{C}\right)$, reduced $\mathrm{pH}(\mathrm{pH} 6.8)$, and increased spirochete cell density (bacterial replication in response to blood nutrients) all are known to activate the RpoN-RpoS pathway [9,21,38-40]. However, inasmuch as these types of shifts in environmental conditions represent artificial in vitro manipulations that cannot fully mimic the spirochete's natural habitats $[37,41,42]$, there may be other aspects of RpoN-RpoS pathway activation that have not yet been appreciated using such in vitro culture conditions as surrogates for natural stimuli. In an attempt to garner more biologically relevant gene expression information and to determine at what specific phase(s) of the enzootic life cycle of B. burgdorferi the RpoN-RpoS pathway is induced and may remain active, we examined the expression of rpos and selected target genes of RpoS over the entire tickmammalian enzootic life cycle.

\section{Results and discussion}

Although in vitro gene expression data have suggested that the RpoN-RpoS pathway is most robust at the tickmammal transmission interface $[9,17,21,36,38-40,43]$, comprehensive gene expression analysis data to support this contention by assessing actual tick and mammalian tissues have been lacking. Furthermore, heretofore, activation of the pathway over the broader tick-mammalian cycle has not been assessed. To address this dearth of information, we examined the expression of rpoS throughout the complete infectious life cycle of $B$. burgdorferi.

\section{rpoS transcription is activated during tick feeding and remains active during mammalian infection by $B$. burgdorferi}

In vitro, rpoS is markedly induced in spirochetes cultivated under conditions that largely mimic tick engorgement, suggesting that rpoS expression is robust during the early transmission phase. Herein, our qRT-PCR analyses indicated that, in larval ticks during acquisition, only 0.4 copies of $r p o S$ transcripts per 100 flaB transcripts were detected in fed larvae, and no rpoS transcripts were detected in intermolt larvae (Figure 1A). However, when exposed to a blood meal, rpoS transcription was dramatically induced; in nymphal ticks following 24,48 , or 72 hours of feeding, $1.8,3.4$, or 8.2 copies of rpoS transcripts per 100 flaB transcripts were detected, respectively (Figure 1A). These data suggest that RpoS is synthesized actively during nymphal tick feeding, and that RpoS then likely transcribes its gene targets. Previously, Caimano et al. [17] reported an increase in rpoS transcripts in engorged infected nymphs (collected at 6-8 days post feeding to repletion). Our more recent data not only are consistent with the findings of Caimano et al. [17], but further pinpoint that the activation of rpoS expression occurs initially in nymphal ticks upon blood feeding.

The cultivation of virulent $B$. burgdorferi in dialysis membrane chambers (DMCs) implanted into the peritoneal cavities of rats has been widely used a surrogate system for studying selected aspects of mammalian infection by B. burgdorferi [41]. However, although previous studies indicated that rpoS transcription was induced when $B$. burgdorferi was cultivated within rat DMCs [17], that approach represents a single temporal sampling that does not take into account disseminatory events that occur during natural mammalian infection. To better address this, we assessed rpoS transcription in mouse tissues at various times post-infection of mice via intradermal needle injection. rpoS transcripts were 


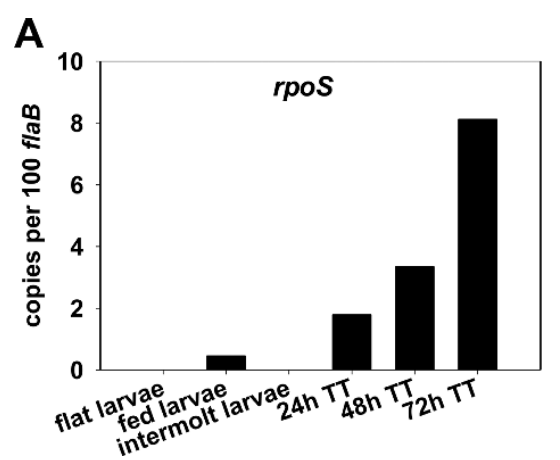

B

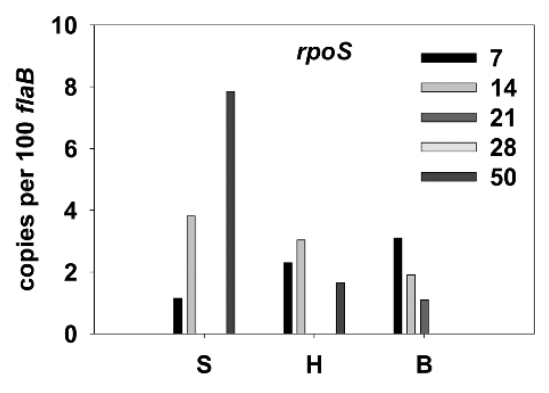

Figure 1 qRT-PCR analysis of rpos transcription in ticks and in mouse tissues. A, flat (uninfected) larvae, fed larvae, intermolt larvae, and fed nymphs during transmission phase were collected at 24-, 48-, and 72-h post-feeding. TT: tick transmission. B, mouse tissues of skin (S) heart $(\mathrm{H})$, and bladder (B) were collected at various numbers of days (inset) after infection. The values represent the average copy number normalized per 100 copies of $B$. burgdorferi flaB transcripts.

readily detected in mouse tissues including skin, heart, and bladder at 7-, 14-, 21-, 28-, and 50-days post-infection (Figure 1B), suggesting that the RpoN-RpoS pathway is active during later disseminatory events of mammalian infection. To our knowledge, these are the first data indicating directly that activation of the RpoNRpoS pathway is sustained throughout early and later phases of the mammalian infection process by $B$. burgdorferi.

Expression of ospC, an RpoS-dependent gene, during tick and mouse infections

Given the importance of OspC to the biology of B. burgdorferi infection $[9,13-15,44,45]$, and the fact that $\operatorname{ssp} C$ is a target of RpoS-mediated transcription [17,19,21,46,47], ospC expression was assessed as a downstream marker of RpoN-RpoS activation. Transcription of $o s p C$ was barely detected in ticks during the acquisition phase (Figure $2 \mathrm{~A})$. However, in engorged nymphal ticks, ospC transcription was dramatically increased, which occurred in concert with rpoS transcription; at 24-, 48-, or 72-h after tick feeding, 35, 46 or 216 copies of ospC per $100 \mathrm{flaB}$ transcripts, respectively, were detected (Figure 2A). These mRNA analyses are consistent with previous studies assessing OspC protein synthesis [7-9] and provide further evidence for the importance of OspC as an early factor critical for B. burgdorferi transmission from its tick vector to a mammalian host.

We further examined osp $C$ transcription within various mouse tissues. Although high levels of osp C transcription were detected in skin, heart, and bladder samples isolated from infected mice at 7-days post infection, $\operatorname{sp} p$ C expression over the course of 50 days postinfection diminished markedly over time in these samples (Figure 2B). In fact, ospC transcripts could not be detected in mouse tissues at 28- and 50-d post-infection (Figure 2B). These data suggest that $\operatorname{sspC}$ transcription is active at the early phase of mammalian infection, but is repressed at the later phases, which is consistent with previous observations made in other studies $[15,48,49]$.

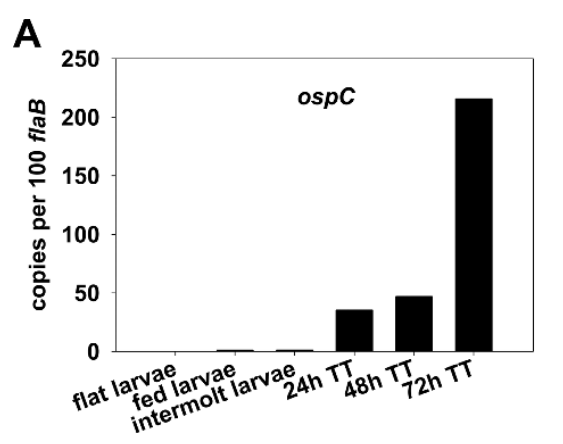

B

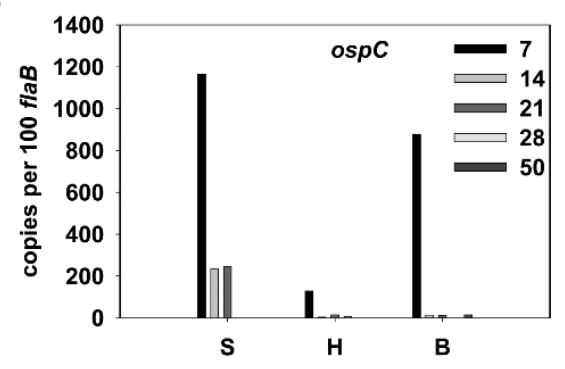

Figure 2 qRT-PCR analysis of $\operatorname{ospC}$ transcription in ticks and in mouse tissues. A, flat (uninfected) larvae, fed larvae, intermolt larvae, and fed nymphs during transmission phase were collected at 24-, 48-, and 72-h post-feeding. TT: tick transmission. B, mouse tissues of skin (S) heart $(H)$, and bladder (B) were collected at various numbers of days (inset) after infection. The values represent the average copy number normalized per 100 copies of B. burgdorferi flaB transcripts. 
Expression of ospA during tick and mouse infections Unlike RpoS-dependent ospC, osp $A$ transcription is believed to be promoted by the housekeeping $\sigma^{70}$-RNA polymerase, through a $\sigma^{70}$-dependent promoter [50]. However, during mammalian infection, osp $A$ also has been shown to be repressed in an RpoS-dependent manner [43], ostensibly via a direct or indirect mechanism. Hodzic et al. [51] also reported that ospA mRNA transcription in the mammalian host is regulated by nonspecific immunoglobulin. Nonetheless, given the well-documented differential regulation pattern of $o s p A$ and $o s p C$ expression, and the dominant role for OspA in B. burgdorferi colonization of the tick midgut, we examined the transcription of ospA throughout the tick-mammalian cycle. Consistent with previous reports examining OspA protein or mRNA [4,7-9,37], ospA was abundantly expressed in ticks during acquisition (Figure 3A); approximately 300 or 210 copies of ospA per $100 \mathrm{flaB}$ transcripts were detected in fed larvae or in intermolt larvae, respectively. However, we also surprisingly observed a considerable increase in $o s p A$ transcription in nymphal ticks during feeding. Approximately 48, 110 , or 380 copies of $o s p A$ per $100 \mathrm{flaB}$ transcripts were detected in nymphal ticks after 24-, 48-, or 72-h of feeding (Figure 3A). It is noteworthy that there have been other reports showing that spirochetes in fed nymphs express both the OspC and OspA lipoproteins simultaneously [7-9,52]. Our transcriptional data regarding ospA/ospC in ticks, in conjunction with the findings of others [7-9,37,52], imply that key mechanistic aspects of the $\operatorname{sp} A$ /ospC regulation paradigm remain to be more fully understood at both the transcriptional and translational levels.

In the majority of mouse skin, heart, and bladder samples, we were unable to detect osp $A$ transcripts (Figure $3 \mathrm{~B})$, suggesting that osp $A$ is not expressed at any appreciable levels during mammalian infection. Previous studies $[53,54]$ noted that antibodies against OspA occur early in human Lyme disease, but diminish with the progress of the disease, hinting that OspA might be expressed by $B$. burgdorferi only during early mammalian infection. Consistent with this, transcripts of osp $A$ were detected in mouse skin samples at 7- or 14- days post-infection (Figure $3 \mathrm{~B}$ ), although the absolute values of $\operatorname{ssp} A$ transcripts were much lower than those for $o s p C$ or $d b p A$ (Figures 2B and $4 \mathrm{~B})$. Our data are in agreement with previous reports by Hodzic et al. [5,51], Liang et al. [55], and Xu et al. [56] who also observed low transcription levels of ospA during murine infection. Of note, this low level of ospA transcription during the early infection phase of needle-inoculated mice may have been influenced by the experimental methodology employed in this study; antibodies to OspA have been detected relatively early upon needle-inoculation of mice with $B$. burgdorferi, but not in mice infected via natural tick bite [51,57]. Nonetheless, the lack of osp $A$ expression during mammalian infection may be due to the presumed RpoS-dependent [43] or immunoglobin-regulated [51] repression of osp $A$ in B. burgdorferi during mammalian infection, and may involve two recently identified putative regulatory elements flanking the ospA promoter [56]. Paradoxically, antibody responses to OspA also have been observed late in the course of human Lyme disease $[51,53,58,59]$, suggesting that $B$. burgdorferi might express OspA again at later stages of infection, perhaps via an unknown regulatory mechanism(s) that overcomes the direct or indirect repression of ospA by RpoS or immunoglobin. Nonetheless, our results revealed that osp $A$ is highly expressed in ticks but is essentially repressed in the early mammalian phase of infection, providing further evidence for the importance of OspA in the biology of $B$. burgdorferi in ticks.

\section{Expression of $d b p A$ throughout the mouse-tick infectious cycles}

In addition to OspC and OspA, other lipoproteins of $B$. burgdorferi also appear to be differentially regulated by

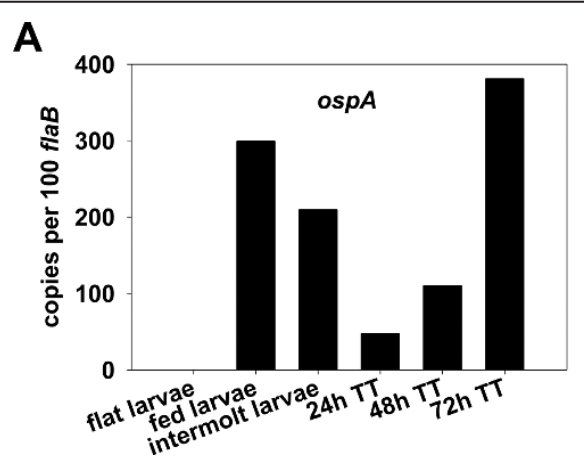

\section{B}

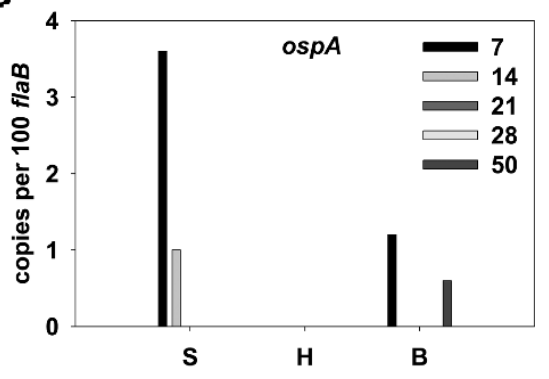

Figure 3 qRT-PCR analysis of ospA transcription in ticks and in mouse tissues. A, flat (uninfected) larvae, fed larvae, intermolt larvae, and fed nymphs during transmission phase were collected at 24-, 48-, and 72-h post-feeding. TT: tick transmission. B, mouse tissues of skin (S) heart $(\mathrm{H})$, and bladder (B) were collected at various numbers of days (inset) after infection. The values represent the average copy number normalized per 100 copies of B. burgdorferi flaB transcripts. 


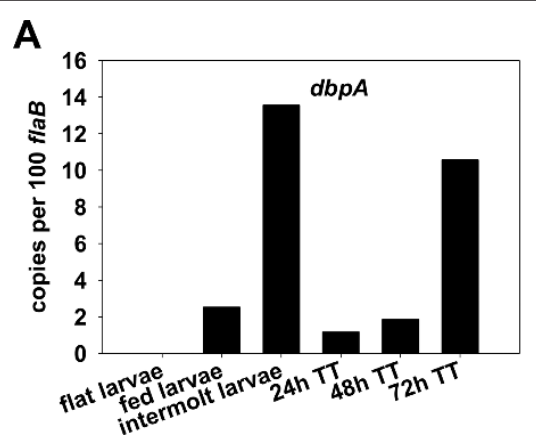

B

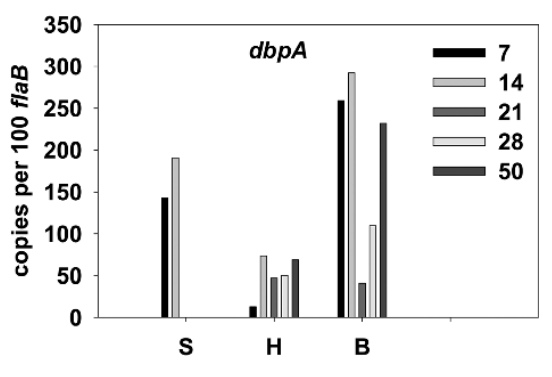

Figure 4 qRT-PCR analysis of $d b p A$ transcription in ticks and in mouse tissues. A, flat (uninfected) larvae, fed larvae, intermolt larvae, and fed nymphs during transmission phase were collected at 24-, 48-, and 72-h post-feeding. TT: tick transmission. B, mouse tissues of skin (S) heart $(\mathrm{H})$, and bladder (B) were collected at various numbers of days (inset) after infection. The values represent the average copy number normalized per 100 copies of B. burgdorferi flaB transcripts.

the RpoN-RpoS pathway in response to varying environmental growth conditions. For example, decorin-binding proteins (DBPs) A and B, presumably serving as adhesins to facilitate the adherence of B. burgdorferi to extracellular matrix as the spirochete invades mammalian tissue, also play important roles in B. burgdorferi infection[60-65]. Mutations in $d b p B A$ lead to a substantial (several $\log$ ) attenuation of $B$. burgdorferi virulence. Previous studies have shown that $B$. burgdorferi alters the expression of $\mathrm{DbpB} / \mathrm{A}$ lipoproteins in response to various environmental factors such as temperature, $\mathrm{pH}$, and spirochetal cell density, influenced largely, if not principally, by the RpoN-RpoS regulatory pathway $[16,19,21,40,66]$. However, although both OspC and DbpA exhibit similar patterns of gene expression when $B$. burgdorferi is cultivated in vitro, there is also abundant evidence that $d b p A$ has an expression pattern slightly different from that of $\operatorname{osp} C$ when $B$. burgdorferi resides in its native environment(s). For example, expression of OspC, but not DbpA, was observed in fed ticks, suggesting a possible suppression mechanism that dampens $d b p B A$ expression in fed ticks [63]. Moreover, ospC expression has been reported to be down-regulated in later phases of mammalian infection, perhaps through a repression mechanism, whereas $d b p A$ expression remains active during the entire phase of mammalian infection $[48,49,63]$. We thus sought to determine whether these differences between osp $C$ and $d b p B A$ expression could be observed via our experimental approach. As shown in Figure 4A, in parallel with rpoS (Figure 1A) and $\operatorname{sspC}$ (Figure 2A) transcription, transcription of $d b p A$ was also induced in nymphal ticks during feeding. $d b p A$ transcripts also were detected in fed larvae and intermolt larvae (Figure 4A) when ospC (Figure 2A) and rpoS transcription (Figure 1A) was essentially absent. There are at least three implications emanating from these findings. First, the results counter those of Hagman et al. [63] wherein the presence of
DbpA lipoprotein was assessed by examining intact borrelia via indirect immunofluorescence; in the current study, $d b p A$ mRNA transcript levels were assessed via more sensitive qRT-PCR. As such, it is difficult to interpret our PCR results in the context of how they may relate to DbpA lipoprotein abundance. Second, a posttranscriptional regulatory mechanism(s) may exist to influence the stability of the mRNA or DbpA protein, which may lead to the suppression of DbpA lipoprotein expression in ticks. Third, given the similarity between RpoS-dependent promoters and $\sigma^{70}$-dependent promoters $[46,67,68]$, our observation that transcription of $d b p A$, but not rpoS, occurred in fed larvae and intermolt larvae also suggests that, unlike ospC, $d b p A$ expression is not entirely dependent on RpoS; transcription of $d b p A$ may also be driven by the housekeeping $\sigma^{70}$ in ticks. Such $\sigma^{70}$-driven $d b p B A$ transcription was not detected within in vitro-grown spirochetes; when $B$. burgdorferi was cultivated in $\mathrm{BSK}$ medium at $37^{\circ} \mathrm{C}$, transcription of $d b p B A$ is essentially dependent on RpoS [66]. This in vitro and in vivo gene expression difference suggests the involvement of potential additional control mechanism(s) in $d b p B A$ transcriptional regulation. Previously, two inverted repeats (IRs) were detected in the 5 'regulatory region of $d b p B A$ [66]. Although these two IRs were not required for the in vitro regulation of $d b p B A$ expression, they may be involved in the activation of $\sigma^{70}$-dependent $d b p B A$ transcription in fed larvae and in intermolt larvae. The binding of a potential trans-activator(s) to these two IRs may be required to facilitate the recruitment of $\sigma^{70}$-RNA polymerase to the $d b p B A$ promoter. Given the lack of $d b p A$ transcription in unfed larvae, such a trans-activator may be expressed by $B$. burgdorferi in fed larvae and intermolt larvae, and the activation of $\sigma^{70}$-dependent $d b p B A$ transcription by a specific regulatory protein may first require some cofactor(s) or ligands contained in mammalian blood. When such a co-factor(s) is depleted with the complete 
digestion of mammalian blood, $d b p A$ transcription may be down-regulated. In nymphs taking a blood meal, the expression of RpoS is highly induced, and then this global regulator, rather than the housekeeping $\sigma^{70}$, likely transcribes $d b p A$. Additional studies are warranted to further elucidate the fine tuning of $d b p B A$ expression, including the putative roles of the IRs in $d b p B A$ gene expression in ticks.

Our data also revealed that $d b p A$ transcripts were readily detected in mouse tissues at all times post-infection, including 7-, 14-, 21-, 28-, and 50-d (Figure 4B), suggesting that $d b p A$ expression remains active during the entire mammalian phase of $B$. burgdorferi infection. These results are fully consistent with other reports using protein detection methods for Dbp assessment [63]. The finding that expression of both rpos and $\operatorname{dbp} A$, but not $\operatorname{sp} C$, in the later phases of mammalian infection also is in agreement with a previous hypothesis [49] that repression of $\operatorname{ssp} C$ may be mediated by a potential trans-acting repressor.

\section{Conclusions}

Since its initial discovery by Hubner et al. [19], the RpoN-RpoS pathway has been the subject of numerous studies seeking to understand core elements of regulatory control in B. burgdorferi [16-18,20-33,37,43, $47,49,52,56,66]$. What has emanated from this expanding body of work is that although certain aspects of the pathway's activation have been predictable, many emerging details have been counter intuitive. One of the unanticipated findings includes the discovery that BosR serves as an additional molecule essential for activation of the RpoN-RpoS pathway [28-31]. In this current study, we again obtained both anticipated and unanticipated experimental results surrounding the activation of the RpoN-RpoS pathway in ticks and during B. burgdorferi dissemination in mammalian tissues.

Our data indicate that the transcription levels of ospC, $d b p A, \operatorname{ssp} A$, or $r p o S$ were variable among mouse samples at different times post-infection. One potential explanation for this is that these important genes are indeed transcribed at different levels within these tissues. Alternatively, it is also possible that our results emanated from low spirochete burdens in these tissue samples, as indicated by the relatively low levels of flaB transcripts detected in these same samples (data not shown). Indeed, the low numbers of spirochetes in certain mouse tissue samples limited our cDNA yields. In order to thus obtain sufficient cDNA for examining gene expression, we were confined to isolating RNA from pooled tissue samples harvested from groups of infected mice, rather than from individual animals. Whereas selective amplification of B. burgdorferi RNA $[69,70]$ potentially may be able to circumvent potential sensitivity limitations in these approaches, such amplification techniques may also incorporate inadvertent bias.

Despite the caveats noted above, some key conclusions regarding activation of the RpoN-RpoS pathway can be drawn from our data. By comparing gene transcription data in ticks during acquisition (fed larvae, intermolt larvae), and in ticks during transmission (nymphal ticks during feeding), the RpoN-RpoS pathway is relatively quiescent in ticks during acquisition, but is initially activated and sustained in nymphs upon feeding. Similar to previous studies [17,37], we assessed gene transcription by isolating RNA from whole ticks, which prevented temporal and spatial analyses of gene expression in specific tick organs. In the future, by using dissected tick organs, gene expression in nymphal midguts and salivary glands at various times during tick feeding may be instructive for discerning how B. burgdorferi exploits the RpoN-RpoS pathway during its migration from midguts to salivary glands and subsequent entry into mammalian tissue. Some unknown factors from mammalian blood also may play critical roles in the induction of this regulatory pathway. Finally, our data demonstrate that the RpoN-RpoS pathway remains relatively active throughout the entire mammalian phase of infection. These combined findings provide further evidence for the central role of the RpoN-RpoS pathway, and its regulated genes, at the interface of B. burgdorferi transmission from tick to mammals and in the establishment of infection in animal hosts.

\section{Methods}

\section{Bacterial strains and growth conditions}

Infectious, low passage (less than 3 passages) B. burgdorferi strain B31 was used throughout this study. B. burgdorferi was routinely cultured in either BSK-II medium or BSK-H medium (Sigma, St. Louis, MO) supplemented with 6\% rabbit serum (Pel-Freeze, Rogers, AR) [71]. Spirochetes were enumerated by dark-field microscopy.

\section{Infection of mice and ticks by $B$. burgdorferi}

All animal experiments were performed according to the protocols approved by the Institutional Animal Care and Use Committee (IACUC) at UT Southwestern Medical Center, Yale University, or the University of Maryland, College Park. To assess activation of the RpoN-RpoS pathway during mammalian infection, adult (4-6 weeks old) female $\mathrm{C} 3 \mathrm{H} / \mathrm{HeN}$ mice were purchased from Charles River laboratories (USA) and were infected with mid-logarithmic phase $B$. burgdorferi via intradermal needle injection $\left(10^{5}\right.$ spirochetes per mouse) at the chest. Spirochetal infection was confirmed by PCR and culture [70]. On days $7,14,21,28$, or 50 post-infection, mice were sacrificed and tissues including heart, joints, bladder, and $7 \mathrm{~mm}$ of skin (harvested from the shaved 
Table 1 Oligonucleotide primers used in this study

\begin{tabular}{|c|c|c|}
\hline Gene & Forward $\left(5^{\prime}-3^{\prime}\right)$ & Reverse $\left(5^{\prime}-3^{\prime}\right)$ \\
\hline \multicolumn{3}{|l|}{ Cloning } \\
\hline$f l a B$ & GGGAACTTGATTAGCCTGCGCAAT & TCGAGCTTCTGATGATGCTGCT \\
\hline rpos & CTTGCAGGACAAATACAAAGAGGC & TGGGACTATTGTCCAGGTTATATCTIT \\
\hline ospC & CTGCTGATGAGTCTGTTAAAGGGC & TाTGGACTTCTGCCACAACAGGG \\
\hline ospA & GCGTTCAGTAGATTGGCTGGTG & CCCTCTAATTTGGTGCCATTTGAGTCG \\
\hline$d b p A$ & CTTATATCATGTGGACTAACAGGAGC & AGCACTCCTTGAGCTGTAGTTGGA \\
\hline \multicolumn{3}{|l|}{ qRT-PCR } \\
\hline flaB & TGATTAGCCTGCGCAATCATT & AATGACAGATGAGGTTGTAGCAGC \\
\hline rpos & CTGGACAAAGAAATAGAGGGATCTG & CAAGGGTAATTTCAGGGTTAAAAGAA \\
\hline ospC & GTACTAAAACTAAAGGTGCTGAAGAA & GCATCTCTITAGCTGCTITTGACA \\
\hline ospA & GGCGTAAAAGCTGACAAAAGTAAAGT & TGTITTGCCATCTTCTITGAAAAC \\
\hline$d b p A$ & ACGAAGCGCTAAAGACATTACAGA & GGCATCAAAATTTACGCCCTTA \\
\hline
\end{tabular}

mouse ventral abdominal region) were harvested and immediately frozen in liquid nitrogen. Frozen samples were stored at $-80^{\circ} \mathrm{C}$ until RNA was isolated.

To prepare B. burgdorferi-infected I. scapularis ticks (representing the tick acquisition phase), mice first were infected intradermally with $B$. burgdorferi B31 $\left(10^{5}\right.$ spirochetes per mouse). After 2 weeks of infection, larvae were fed on animals ( 100 larvae per mouse) and approximately 50 fed ticks were collected for RNA isolation. The other 50 fed larvae were allowed to remain in an incubator for a period of 3 weeks, and 25 ticks were collected as fed intermolt larvae. Remaining fed larval ticks were allowed to molt to nymphs. Newly molted unfed infected nymphs were then allowed to feed on naïve mice ( 25 ticks per mouse) (tick transmission phase). The nymphs were collected at 24,48 , or $72 \mathrm{~h}$ post-infestation and stored in liquid nitrogen until processed for RNA extraction. As a control, flat larvae were also collected for RNA extraction and subsequent gene expression analysis.

\section{RNA extraction and CDNA synthesis}

Total RNA was isolated from mice and tick samples as previously described [70,72]. Briefly, frozen mouse bladder, heart, joints, and skin samples $(\sim 30 \mathrm{mg})$ were thoroughly ground using mortar and pestle in the presence of liquid nitrogen and immediately transferred to precooled eppendorf tubes containing RLT buffer (Qiagen RNeasy Mini kit, Qiagen, CA). Samples were then passed through a syringe fitted with a 18-1/2 gauge needle several times on ice to make a homogeneous suspension and were then processed for total RNA extraction using RNeasy Mini kit (Qiagen) following the manufacturer's instructions. Total RNA was isolated from whole tick samples by using the TRIzol reagent (Invitrogen, Carlsbad, CA) and further purified as described by the manufacturer in the accessory protocol for cleanup of RNA using the RNeasy Mini kit (Qiagen).
Genomic DNA was removed from all RNA preparations by using Turbo DNAfree (Ambion, Austin, TX) and verified by PCR analysis. cDNA was synthesized using the BioRad iScript cDNA synthesis kit (BioRad, Hercules, CA) according to the manufacturer's instructions. Of note, despite several attempts, cDNA yields from mouse joint samples were inadequate for examining gene expression, likely due to low spirochete burdens in these samples. Nonetheless, we were able to obtain sufficient cDNA from other mouse samples (including skin, heart, and bladder) and infected ticks for gene expression analyses.

\section{Quantitative RT-PCR analysis}

Quantitative PCR (qPCR) using the Platinum SYBR Green qPCR SuperMix-UDG kit (Invitrogen) was employed to measure amplicons present in mouse and tick cDNA samples. Specific primers (Table 1) for $B$. burgdorferi genes flaB, rpoS, ospC, $d b p A$, and $\operatorname{sp} A$, were designed by using PRIMEREXPRESS software (Applied Biosystems, Carlsbad, CA) and validated by using 10fold dilutions (10-0.0000001 ng) of B. burgdorferi genomic DNA in an absolute quantification test on an ABI 7500 real-time PCR system (Applied Biosystems). Standard curves created for all primers had a slope of $-3.3 \pm$ 0.3 (data not shown). For quantification of amplicons, an individual gene was first amplified by PCR and cloned into the pGEM-Teasy vector (Promega, Madison, WI). Recombinant plasmid DNA was then purified and diluted serially 10 -fold to generate a standard curve. Transcript copies corresponding to each gene of interest were calculated using the Absolute Quantification Analysis program (Applied Biosystems) and normalized against copies of flaB.

\section{Acknowledgements}

We thank Jianli Zhou for technical assistance. This work was supported by NIH-NIAID Grants Al-059062 (to MVN), Al-076705 (to SN) and Al-080615 (to 
UP), an Arthritis Foundation fellowship (to GN), and an award from the American Heart Association (to ZO).

\section{Author details}

'Department of Microbiology, University of Texas Southwestern Medica Center, 5323 Harry Hines Blvd, Dallas, TX 75390, USA. ${ }^{2}$ Section of Infectious Diseases, Department of Internal Medicine, Yale University School of Medicine, New Haven, CT 06520, USA. ${ }^{3}$ Department of Veterinary Medicine, University of Maryland, College Park, MD 20742, USA.

\section{Authors' contributions}

ZO, SN, GN, and MK performed experiments. ZO and MVN analyzed results. ZO, UP, EF and MVN participated in experimental designs and writing of the manuscript. All authors read and approved the manuscript.

Received: 13 January 2012 Accepted: 23 March 2012 Published: 23 March 2012

\section{References}

1. Bacon RM, Kugeler KJ, Mead PS: Surveillance for Lyme disease-United States, 1992-2006. MMWR Surveill Summ 2008, 57(10):1-9.

2. Burgdorfer W, Barbour AG, Hayes SF, Benach JL, Grunwaldt E, Davis JP. Lyme disease-a tick-borne spirochetosis? Science 1982, 216(4552):1317-1319.

3. Steere AC, Grodzicki RL, Kornblatt AN, Craft JE, Barbour AG, Burgdorfer W, Schmid GP, Johnson E, Malawista SE: The spirochetal etiology of Lyme disease. N Engl J Med 1983, 308(13):733-740.

4. de Silva AM, Telford SR, Brunet LR, Barthold SW, Fikrig E: Borrelia burgdorferi OspA is an arthropod-specific transmission-blocking Lyme disease vaccine. J Exp Med 1996, 183(1):271-275.

5. Hodzic E, Feng S, Freet KJ, Borjesson DL, Barthold SW: Borrelia burgdorferi population kinetics and selected gene expression at the host-vector interface. Infect Immun 2002, 70(7):3382-3388.

6. Montgomery RR, Malawista SE, Feen KJ, Bockenstedt LK: Direct demonstration of antigenic substitution of Borrelia burgdorferi ex vivo: exploration of the paradox of the early immune response to outer surface proteins A and C in Lyme disease. J Exp Med 1996, 183(1):261-269.

7. Ohnishi J, Piesman J, de Silva AM: Antigenic and genetic heterogeneity of Borrelia burgdorferi populations transmitted by ticks. Proc Natl Acad Sci USA 2001, 98(2):670-675.

8. Schwan TG, Piesman J: Temporal changes in outer surface proteins $A$ and $\mathrm{C}$ of the lyme disease-associated spirochete, Borrelia burgdorferi, during the chain of infection in ticks and mice. J Clin Microbiol 2000, 38(1):382-388.

9. Schwan TG, Piesman J, Golde WT, Dolan MC, Rosa PA: Induction of an outer surface protein on Borrelia burgdorferi during tick feeding. Proc Natl Acad Sci USA 1995, 92(7):2909-2913.

10. Pal U, de Silva AM, Montgomery RR, Fish D, Anguita J, Anderson JF, Lobet Y, Fikrig E: Attachment of Borrelia burgdorferi within Ixodes scapularis mediated by outer surface protein A. J Clin Invest 2000, 106(4):561-569.

11. Pal U, Li X, Wang T, Montgomery RR, Ramamoorthi N, Desilva AM, Bao F, Yang X, Pypaert M, Pradhan D, et al: TROSPA, an Ixodes scapularis receptor for Borrelia burgdorferi. Cell 2004, 119(4):457-468.

12. Yang XF, Pal U, Alani SM, Fikrig E, Norgard MV: Essential role for OspA/B in the life cycle of the Lyme disease spirochete. J Exp Med 2004, 199(5):641-648.

13. Grimm D, Tilly K, Byram R, Stewart PE, Krum JG, Bueschel DM, Schwan TG, Policastro PF, Elias AF, Rosa PA: Outer-surface protein C of the Lyme disease spirochete: a protein induced in ticks for infection of mammals. Proc Natl Acad Sci USA 2004, 101(9):3142-3147.

14. Pal U, Yang X, Chen M, Bockenstedt LK, Anderson JF, Flavell RA, Norgard MV, Fikrig E: OspC facilitates Borrelia burgdorferi invasion of Ixodes scapularis salivary glands. J Clin Invest 2004, 113(2):220-230.

15. Tilly K, Krum JG, Bestor A, Jewett MW, Grimm D, Bueschel D, Byram R, Dorward D, Vanraden MJ, Stewart P, et al: Borrelia burgdorferi OspC protein required exclusively in a crucial early stage of mammalian infection. Infect Immun 2006, 74(6):3554-3564.

16. Caimano MJ, Eggers $\mathrm{CH}$, Hazlett KR, Radolf JD: RpoS is not central to the general stress response in Borrelia burgdorferi but does control expression of one or more essential virulence determinants. Infect Immun 2004, 72(11):6433-6445.

17. Caimano MJ, lyer R, Eggers CH, Gonzalez C, Morton EA, Gilbert MA, Schwartz I, Radolf JD: Analysis of the RpoS regulon in Borrelia burgdorferi in response to mammalian host signals provides insight into RpoS function during the enzootic cycle. Mol Microbiol 2007, 65(5):1193-1217.

18. Fisher MA, Grimm D, Henion AK, Elias AF, Stewart PE, Rosa PA, Gherardini FC: Borrelia burgdorferi sigma54 is required for mammalian infection and vector transmission but not for tick colonization. Proc Nat/ Acad Sci USA 2005, 102(14):5162-5167.

19. Hubner A, Yang X, Nolen DM, Popova TG, Cabello FC, Norgard MV: Expression of Borrelia burgdorferi OspC and DbpA is controlled by a RpoN-RpoS regulatory pathway. Proc Natl Acad Sci USA 2001, 98(22):12724-12729.

20. Smith AH, Blevins JS, Bachlani GN, Yang XF, Norgard MV: Evidence that RpoS (sigmaS) in Borrelia burgdorferi is controlled directly by RpoN (sigma54/sigmaN). J Bacteriol 2007, 189(5):2139-2144.

21. Samuels DS: Gene regulation in Borrelia burgdorferi. Annu Rev Microbiol 2011, 65:479-499.

22. Boardman BK, He M, Ouyang Z, Xu H, Pang X, Yang XF: Essential role of the response regulator Rrp2 in the infectious cycle of Borrelia burgdorferi. Infect Immun 2008, 76(9):3844-3853.

23. Burtnick MN, Downey JS, Brett PJ, Boylan JA, Frye JG, Hoover TR, Gherardini FC: Insights into the complex regulation of rpoS in Borrelia burgdorferi. Mol Microbiol 2007, 65(2):277-293.

24. Ouyang Z, Blevins JS, Norgard MV: Transcriptional interplay among the regulators Rrp2, RpoN and RpoS in Borrelia burgdorferi. Microbiology 2008, 154(Pt 9):2641-2658.

25. Xu H, Caimano MJ, Lin T, He M, Radolf JD, Norris SJ, Gherardini F, Wolfe AJ, Yang XF: Role of acetyl-phosphate in activation of the Rrp2-RpoN-RpoS pathway in Borrelia burgdorferi. PLoS Pathog 2010, 6(9):e1001104.

26. Yang XF, Alani SM, Norgard MV: The response regulator Rrp2 is essential for the expression of major membrane lipoproteins in Borrelia burgdorferi. Proc Natl Acad Sci USA 2003, 100(19):11001-11006.

27. Blevins JS, Xu H, He M, Norgard MV, Reitzer L, Yang XF: Rrp2, a sigma54dependent transcriptional activator of Borrelia burgdorferi, activates rpoS in an enhancer-independent manner. J Bacteriol 2009, 191(8):2902-2905.

28. Hyde JA, Shaw DK, Smith lii R, Trzeciakowski JP, Skare JT: The BosR regulatory protein of Borrelia burgdorferi interfaces with the RpoS regulatory pathway and modulates both the oxidative stress response and pathogenic properties of the Lyme disease spirochete. Mol Microbiol 2009, 74(6):1344-1355.

29. Ouyang Z, Kumar M, Kariu T, Haq S, Goldberg M, Pal U, Norgard MV: BosR (BB0647) governs virulence expression in Borrelia burgdorferi. Mol Microbiol 2009, 74(6):1331-1343.

30. Ouyang Z, Deka RK, Norgard MV: BosR (BB0647) controls the RpoN-RpoS regulatory pathway and virulence expression in Borrelia burgdorferi by a novel DNA-binding mechanism. PLoS Pathog 2011, 7(2):e1001272.

31. Samuels DS, Radolf JD: Who is the BosR around here anyway? $\mathrm{Mol}$ Microbiol 2009, 74(6):1295-1299.

32. Lybecker MC, Abel CA, Feig AL, Samuels DS: Identification and function of the RNA chaperone Hfq in the Lyme disease spirochete Borrelia burgdorferi. Mol Microbiol 2010, 78(3):622-635.

33. Lybecker MC, Samuels DS: Temperature-induced regulation of RpoS by a small RNA in Borrelia burgdorferi. Mol Microbiol 2007, 64(4):1075-1089.

34. Karna SL, Sanjuan E, Esteve-Gassent MD, Miller CL, Maruskova M, Seshu J: CsrA modulates levels of lipoproteins and key regulators of gene expression critical for pathogenic mechanisms of Borrelia burgdorferi. Infect Immun 2011, 79(2):732-744.

35. Sze CW, Morado DR, Liu J, Charon NW, Xu H, Li C: Carbon storage regulator $\mathrm{A}(\mathrm{Csr} \mathrm{A}(\mathrm{Bb}))$ is a repressor of Borrelia burgdorferi flagellin protein FlaB. Mol Microbiol 2011, 82(4):851-864.

36. Brooks CS, Hefty PS, Jolliff SE, Akins DR: Global analysis of Borrelia burgdorferi genes regulated by mammalian host-specific signals. Infect Immun 2003, 71(6):3371-3383.

37. Mulay VB, Caimano MJ, lyer R, Dunham-Ems S, Liveris D, Petzke MM, Schwartz I, Radolf JD: Borrelia burgdorferi bba74 is expressed exclusively during tick feeding and is regulated by both arthropod- and mammalian host-specific signals. J Bacterio/ 2009, 191(8):2783-2794.

38. Tokarz R, Anderton JM, Katona LI, Benach JL: Combined effects of blood and temperature shift on Borrelia burgdorferi gene expression as 
determined by whole genome DNA array. Infect Immun 2004, 72(9):5419-5432.

39. Revel AT, Talaat AM, Norgard MV: DNA microarray analysis of differential gene expression in Borrelia burgdorferi, the Lyme disease spirochete. Proc Natl Acad Sci USA 2002, 99(3):1562-1567.

40. Yang X, Goldberg MS, Popova TG, Schoeler GB, Wikel SK, Hagman KE, Norgard MV: Interdependence of environmental factors influencing reciprocal patterns of gene expression in virulent Borrelia burgdorferi. Mol Microbiol 2000, 37(6):1470-1479.

41. Akins DR, Bourell KW, Caimano MJ, Norgard MV, Radolf JD: A new animal model for studying Lyme disease spirochetes in a mammalian hostadapted state. J Clin Invest 1998, 101(10):2240-2250.

42. Cugini C, Medrano M, Schwan TG, Coburn J: Regulation of expression of the Borrelia burgdorferi beta(3)-chain integrin ligand, P66, in ticks and in culture. Infect Immun 2003, 71(2):1001-1007.

43. Caimano MJ, Eggers CH, Gonzalez CA, Radolf JD: Alternate sigma factor RpoS is required for the in vivo-specific repression of Borrelia burgdorferi plasmid Ip54-borne ospA and Ip6.6 genes. J Bacteriol 2005, 187(22):7845-7852.

44. Ramamoorthi N, Narasimhan S, Pal U, Bao F, Yang XF, Fish D, Anguita J, Norgard MV, Kantor FS, Anderson JF, et al: The Lyme disease agent exploits a tick protein to infect the mammalian host. Nature 2005, 436(7050):573-577.

45. Xu Q, McShan K, Liang FT: Essential protective role attributed to the surface lipoproteins of Borrelia burgdorferi against innate defences. Mol Microbiol 2008, 69(1):15-29.

46. Eggers $\mathrm{CH}$, Caimano MJ, Radolf JD: Analysis of promoter elements involved in the transcriptional initiation of RpoS-dependent Borrelia burgdorferi genes. J Bacteriol 2004, 186(21):7390-7402.

47. Yang XF, Lybecker MC, Pal U, Alani SM, Blevins J, Revel AT, Samuels DS, Norgard MV: Analysis of the $o s p C$ regulatory element controlled by the RpoN-RpoS regulatory pathway in Borrelia burgdorferi. J Bacteriol 2005, 187(14):4822-4829.

48. Liang FT, Jacobs MB, Bowers LC, Philipp MT: An immune evasion mechanism for spirochetal persistence in Lyme borreliosis. J Exp Med 2002, 195(4):415-422

49. Xu Q, McShan K, Liang FT: Identification of an $\operatorname{csp} \mathrm{C}$ operator critical for immune evasion of Borrelia burgdorferi. Mol Microbiol 2007, 64(1):220-231.

50. Sohaskey CD, Zuckert WR, Barbour AG: The extended promoters for two outer membrane lipoprotein genes of Borrelia spp. uniquely include a Trich region. Mol Microbiol 1999, 33(1):41-51.

51. Hodzic E, Tunev S, Feng S, Freet KJ, Barthold SW: Immunoglobulinregulated expression of Borrelia burgdorferi outer surface protein A in vivo. Infect Immun 2005, 73(6):3313-3321.

52. Srivastava SY, de Silva AM: Reciprocal expression of ospA and ospC in single cells of Borrelia burgdorferi. J Bacteriol 2008, 190(10):3429-3433.

53. Kalish RA, Leong JM, Steere AC: Early and late antibody responses to fulllength and truncated constructs of outer surface protein A of Borrelia burgdorferi in Lyme disease. Infect Immun 1995, 63(6):2228-2235.

54. Schutzer SE, Coyle PK, Dunn JJ, Luft BJ, Brunner M: Early and specific antibody response to OspA in Lyme Disease. J Clin Invest 1994, 94(1):454-457.

55. Liang FT, Caimano MJ, Radolf JD, Fikrig E: Borrelia burgdorferi outer surface protein (osp) B expression independent of ospA. Microb Pathog 2004, 37(1):35-40.

56. Xu Q, McShan K, Liang FT: Two regulatory elements required for enhancing ospA expression in Borrelia burgdorferi grown in vitro but repressing its expression during mammalian infection. Microbiology 2010, 156(Pt 7):2194-2204.

57. Gern L, Schaible UE, Simon MM: Mode of inoculation of the Lyme disease agent Borrelia burgdorferi influences infection and immune responses in inbred strains of mice. J Infect Dis 1993, 167(4):971-975.

58. Barbour AG, Burgdorfer W, Grunwaldt E, Steere AC: Antibodies of patients with Lyme disease to components of the Ixodes dammini spirochete. J Clin Invest 1983, 72(2):504-515.

59. Krause A, Burmester GR, Rensing A, Schoerner C, Schaible UE, Simon MM, Herzer $P$, Kramer MD, Wallich R: Cellular immune reactivity to recombinant OspA and flagellin from Borrelia burgdorferi in patients with Lyme borreliosis. Complexity of humoral and cellular immune responses. J Clin Invest 1992, 90(3):1077-1084.
60. Blevins JS, Hagman KE, Norgard MV: Assessment of decorin-binding protein A to the infectivity of Borrelia burgdorferi in the murine models of needle and tick infection. BMC Microbiol 2008, 8:82.

61. Coburn J: Adhesion mechanisms of the Lyme disease spirochete, Borrelia burgdorferi. Curr Drug Targets Infect Disord 2001, 1(2):171-179.

62. Guo BP, Norris SJ, Rosenberg LC, Hook M: Adherence of Borrelia burgdorferi to the proteoglycan decorin. Infect Immun 1995, 63(9):3467-3472.

63. Hagman KE, Yang X, Wikel SK, Schoeler GB, Caimano MJ, Radolf JD, Norgard MV: Decorin-binding protein A (DbpA) of Borrelia burgdorferi is not protective when immunized mice are challenged via tick infestation and correlates with the lack of DbpA expression by B. burgdorferi in ticks. Infect Immun 2000, 68(8):4759-4764.

64. Shi Y, Xu Q, McShan K, Liang FT: Both decorin-binding proteins A and B are critical for overall virulence of Borrelia burgdorferi. Infect Immun 2008, 76:1239-1246.

65. Weening EH, Parveen N, Trzeciakowski JP, Leong JM, Hook M, Skare JT: Borrelia burgdorferi lacking DbpBA exhibits an early survival defect during experimental infection. Infect Immun 2008, 76(12):5694-5705.

66. Ouyang $Z$, Haq S, Norgard MV: Analysis of the $d b p B A$ upstream regulatory region controlled by RpoS in Borrelia burgdorferi. J Bacteriol 2010, 192(7):1965-1974.

67. Becker G, Hengge-Aronis R: What makes an Escherichia coli promoter sigma(S) dependent? Role of the -13/-14 nucleotide promoter positions and region 2.5 of sigma(S). Mol Microbiol 2001, 39(5):1153-1165.

68. Typas A, Becker $G$, Hengge R: The molecular basis of selective promoter activation by the sigmaS subunit of RNA polymerase. Mol Microbiol 2007, 63(5):1296-1306.

69. Narasimhan S, Caimano MJ, Liang FT, Santiago F, Laskowski M, Philipp MT, Pachner AR, Radolf JD, Fikrig E: Borrelia burgdorferi transcriptome in the central nervous system of non-human primates. Proc Natl Acad Sci USA 2003, 100(26):15953-15958

70. Pal U, Wang P, Bao F, Yang X, Samanta S, Schoen R, Wormser GP, Schwartz I, Fikrig E: Borrelia burgdorferi basic membrane proteins A and B participate in the genesis of Lyme arthritis. J Exp Med 2008, 205(1):133-141.

71. Pollack RJ, Telford SR, Spielman A: Standardization of medium for culturing Lyme disease spirochetes. J Clin Microbiol 1993, 31(5):1251-1255.

72. Yang X, Coleman AS, Anguita J, Pal U: A chromosomally encoded virulence factor protects the Lyme disease pathogen against hostadaptive immunity. PLoS Pathog 2009, 5(3):e1000326.

doi:10.1186/1471-2180-12-44

Cite this article as: Ouyang et al: Activation of the RpoN-RpoS regulatory pathway during the enzootic life cycle of Borrelia burgdorferi. BMC Microbiology 2012 12:44.

\section{Submit your next manuscript to BioMed Central and take full advantage of:}

- Convenient online submission

- Thorough peer review

- No space constraints or color figure charges

- Immediate publication on acceptance

- Inclusion in PubMed, CAS, Scopus and Google Scholar

- Research which is freely available for redistribution 